turned Indians. A long residence in the East, without the intervention of fever, may alone induce the irregular state of the pulse now under consideration; but a lengthened stay in India, together with repeated fevers, is almost certain to cause it.

Complicated with all these morbid conditions, alterations in the structure and functions of the mucous digestive surfaces are frequently observable, with their train of irritable dyspepsias and constipations.

In the great majority of persons suffering from the sequelæe of tropical fevers, we find that the whole abdomen is tumid, doughy, and inelastic, with evident venous congestion of all its viscera, while the action of the heart and arteries is feeble. There is, in fact, such a stagnation of the venous blood as to weaken the functions and spoil the secretions of the stomach, liver, bowels, and spleen, while the general circulation, and the functions of the skin in particular, are oppressed and enfeebled.

All these conditions become aggravated when the sufferer from tropical fever arrives in England in the winter or spring seasons. In other instances we find congestive enlargements, with the commencement of interstitial deposits, more or less chronic, of the liver, spleen, and mesentery, accompanied, as in the former case of simple abdominal congestion, by torpor of the digestive powers, depravation of all the secretions, a feeble and oppressed circulation, and marked derangement of the cuticular functions. In both instances there is great sensitiveness to the impression of cold. The medical management of such cases, whether functionally or structurally affected, is always a matter requiring care, and the cure may occasionally, in persons greatly debilitated by fever, or by long residence in India, be protracted. We have to emulge with one hand, while we impart tone with the other. We have to maintain a gentle but persistent action in all the depurative organs, at the same time that by medicine, proper diet, free exposure to the air, and by exercise, we improve the quality and angment the quantity of the blood.

CASE 1.- $\Lambda$ gentleman of robust frame and short stature, aged thirty-eight, had resided sixteen years in India as an indigo planter, and during that time he had been continually, and in all seasons, much exposed to direct solar heats, to excessive rains, and to cold. His habits of life had been moderate, and for fourteen years his health had been excellent. Two years ago, while exposed to cold and damp weather, he was seized with severe intermittent fever, the stage of rigor lasting frequently for three hours. Medical aid was obtained from a distance, and but irregularly. Calomel and antimony, followed by brisk purgatives, and by quinine, overcame the immediate violence of the fever, but he has ever since been out of health. The least exposure to the sun, to damp or cold, - circumstances never considered when in health,-now excited him to fever, especially if such exposure occurred about the full and change of the moon. His fever by degrees, and under the influence of quinine, lost the severity of the rigor, until that symptom entirely ceased, and on the accession of the cold season it assumed the continued form. At length he became worn out by repeated irregular attacks of fever. His muscular system, which had been powerful, fell away, while the abdomen became large, full, and tense. In this state he was recommended to return to England, where he arrived in the month of March.

He now presents the true physconia, or parabysma, of systematic writers, in the sense of " morbid congestion."

Among the causes of this state of stagnation, Dr. Mason Good justly considers the destitution of valves in the abdominal veins as prominent, owing to that want of support to the returning column of blood which belongs to the veins distributed to more superficial parts. That the cold stage of severe intermittent fever, or the stage of congestion, must, when often repeated, tend powerfully to produce, and to maintain, stagnation in the abdominal venous trunks, seems evident. It proved so in this case. The abdomen is now full, hard, and round as a barrel, but percussion affords no evidence of enlargement of either liver or spleen. The complexion is of a copper-yellow hue. The skin is harsh, dry, and cool, and when the integument is pressed between the finger and thumb, the marks are long in regaining the colour of the surrounding parts, owing to the feebleness of the capillary circulation. The sublingual and conjunctival surfaces look pale, and exhibit a deficiency of red particles in the blood; there seems to be an entire suspension of the exhalent and absorbing functions; the pulse is feeble, slow, and oppressed; the respiration hurried on the least exertion, and impeded by the fulness and tension of the abdomen. Abdominal congestion would seem to be the sole cause of his weakness and his sufferings. Tho bowels never act without the aid of medicine; the intestinal secretions are pale and scanty; the urine natural in appear- ance. The muscular frame is so enfeebled that he cannot walk an hundred yards without fatigue. His sleep is broken, uneasy, and unrefreshing.

The patient was directed to take a tablespoonful of the following mixture every morning in a small tumbler of cold water, or so much as should produce two evacuations daily:- Saturated solution of sulphate of magnesia, seven ounces and a half; dilute sulphuric acid, half an ounce; sulphate of iron, sulphate of quinine, of each half a drachm. He was directed at the same time to use two doses daily of a mixture composed of fluid extract of taraxacum with bitartrate of potash. These medicines after a time acted freely on the bowels and kidneys, while the functions of the skin were promoted by warm baths twice $a$ week, immediately before going to bed. Frictions to thesurface were prescribed; and horse exercise advised. The diet was ordered to be light and spare; and as all food produced a sense of weight and oppression, he was allowed to take a small tumbler of weak branty-and-water at dinner. On this plan the patient improved steadily, though slowly, during six weeks, when he wrote to me from the country that he could walk for an hour without fatigue or hurried respiration, his waist being now reduced by seven inches. The biliary secretion became abundant, and the appetite and digestion had greatly improved. The taraxacum was now exchanged for a pure chalybeate, and the purgative was directed to be used every other day only. When the weather became warm in May, the convalescence was rapid; and in the following month he proceeded on a tour to the continent. He used the waters of Homburg; but in his own opinion the change of air and the continued exercise were of most effect. He returned to England in September, in perfect health.

Remarks.-This was a case of excessive abdominal congestion resulting from fever, accompanied by anæmia. This state of the blood was doubtless maintained by the stagnation of so large a portion of the circulating fluid in the abdominal venous trunks. The whole machinery of life was clogged. Youth, a robust constitution, and temperate habits of life, favoured the patient and aided his recovery. His treatment was simple but effective. In a case such as this, a persistent course of tonic purgatives is always necessary; and $I$ believe that purgative medicines, when conjoined with tonics and chalybeates, as in the present instance, do not injure the functions of the colon, as they are too apt to do when given singly in large or repeated doses, or where given with or after calomel.

When this gentleman arrived in England, it was supposed by his medical attendant in the country, that effusion had taken place within the abdominal cavity, but this I was unable to verify, owing to the extreme fulness and tension of the entire region. Certainly the case wore a very unfavourable aspect, and dropsy, general or local, might well have been apprehended.

(To be continued.)

ON THE

\section{ADMINISTRATION OF REMEDIAL AGENTS IN PHTHISIS, IN COMBINATION WITH PROTEINE COMPOUNDS.}

By THOMAS CATTELL, M.D., M.R.C.S., \&c., Braunston, Northamptonshire.

The South-American treatment of phthisis; modes adopted in the administration of remedies; pathological indications of phthisis; value of iodine in the treatment of phthisis; salts of iodine \&c. always to be given in a proteine compound-the value of albumen for this purpose; a system similar to that employed in South America might be adopted in Great Britain; the same might be introduced into lunatic asylums; object to be held in view in the treatment of phthisis; prophylactic treatment, \&c.

There is a quotation in The Lancer of the 26 th April, concerning the plan pursued by Messrs. Jackson, Brothers, entitled "South-American cure for phthisis," which I perceive involves a principle long since adopted by me, in the treatment of the same disease. Either a salt of iodine has been directed to be taken in milk, beef-tea, or solution of albumen; or some compound or combination of iodine to be administered in the usual way, and the diet restricted to milk, the most easily digestible kind of animal food, eggs, gluten, fluid extract of beef, and vegetables containing no starch.

By pursuing this principle of treatment we secure whatever therapeutical advantages may result from that methodus 
medendi which either associates the curative agent with the nutritive aliment destined to become a constituent of the organism, or which restricts the patient to a diet that does not in any way tend to counteract the influence of the remedy.

We ought not to overlook the fact that a lesion of nutrition - general asthenia-is one of the most obvious and unmistakable symptoms which characterize the phthisical diathesisloss of weight, the immediate consequence of the local malady.

The healthy relation between the processes of nutrition and depuration are always more or less impaired, and as the disease advances the impairment becomes fearfully increased.

Iodine ranks deservedly high amongst the remedies best calculated to re-establish a healthy physiological relation between these two great functions of the animal economy: it frequently induces great improvement in phthisical cases, probably by the influence which it exerts over the processes of assimilation and absorption. It is, however, by no means the only remedy to which our attention should be directed: the mercurial pill, mercury in combination with iron and confection of roses, mercurial pill in combination with nitrate of silver \&c., hydriodic acid, bromide of potassium, phosphoric acid, phosphate of lime, and the phosphates of quinine and iron, recently introduced by me to the notice of the profession, may also induce important therapeatic changes.

The salts of iodine, from their activity on the absorbents of the alimentary canal, should seldom or never be employed, unless in a fluid animal proteine compound, or solution. It is after this mode of exhibition that the system tolerates the medicine, unaccompanied by that sensation of exhaustion and sinking so generally experienced, when administered, as the medicine usually is, apart from intermixture with animal organic compounds.

I feel justified in remarking here, that sufficient attention has not been given to the administration of certain remedies in combination with a proteine body. Chemistry and physiology point out to us albumen as the proteine body most likely to prove of the greatest value for this purpose. For some time past I have been testing the efficacy of this method of treatment in various diseases, employing albumen in combination with iron, iodine, and other remedial agents. I purpose hereafter to direct the attention of the profession more fully to the employment of albumen in this way.

To return however to the occasion of these remarks. I may observe, that whatever value may attach to the "South American cure for phthisis," British practitioners cannot have recourse to the roundabout process of feeding llamas on the "fucus iodiferus," with a view of testing the therapeutical value of iodide of sodium in combination with a solid or fluid animal proteine substance; neither is such a course in any way necessary to secure the advantages of this principle of treatment. The caseine, as contained in the milk of the cow, possesses a chemical composition identical with that obtained from the llamas; and the animal food which we daily consume differs not as a proteine compound from that yielded by the same animal.

It is evident, therefore, that by the adoption of the following plan we may secure all the advantages which can possibly result from the South American mode of treatment.

1. Iodide of sodium may be given in cow's milk. There is considerable advantage in this mode of administration, as we can regulate the quantity of the salt of iodine, and estimate its effects.

2. To impregnate animal substances with iodide of sodium, or any other soluble salt, it is merely necessary to employ an instrument constructed on the principle of the force-pump.

The adoption of such methods might occasionally be had recourse to with great advantage in the treatment of lunatics that obstinately refuse every kind of medicament.

In counexion with the medical treatment of phthisis, I would observe that it ought to be a paramount object at all times to prescribe a regimen calculated to produce blood rich in the plastic elements of nutrition.

This suggestion is particularly deserving notice in reference to young persons of a phthisical diathesis; here a greater amount of proteinised compounds are required to supply the material for growth, as well as to maintain the integrity of the organism, than in persons of more advanced years. There is no doubt that the ravages of phthisis among young persons is mainly attributable to an utter neglect of this simple precaution, the lungs in consequence taking on morbid action. It is, however, my intention to return to this important subject at a fitting opportunity.

Braunston, $18 \overline{1} 1$.

\section{REPORT OF \\ A CASE OF ACCIDENTAL POISONING BY TINCTURE OF ACONITE.}

By JOHN TOPHAM, M.D. Lond.,

PHYSICIAN TO THE SOUTH STAFFORDSHIRE GENERAL MOSPITAL, WOLYRRHAMPTON; AND CONSULTING PHYSICIAN TO THE BRDGNORTH INFIRMARY.

A woMan, twenty-seven years of age, labouring under the effects of anæmia, conjoined with leucorrhoea and gastrodynia, had a prescription given her, consisting of citrate of quinine and iron, dissolved in water. The dispenser, owing to some error in compounding, added half an ounce of tincture of aconite to the eight-ounce mixture ordered. At eleven o'clock in the morning, she took one tablespoonful of the above compound, and immediately felt a sensation of numb. ness in the tongue, accompanied by difficulty of swallowing; soon afterwards she began to cry violently, this act being accompanied by convulsive twitchings of the facial muscles. Immediately after this she lost the power of walking, and began to eject a quantity of mucus from the stomach. At half-past two o'clock, I saw her, and observed the following symptoms, which, joined to the details just mentioned, caused me to suspect that aconite had been taken. She was lying upon a sofa, resting upon her back, with her eyelids closed, and the pupils slightly contracted. She was quite unconscious when spoken to, and during an hour's observation she only spoke intelligibly twice, and then said, "What is it?" Every now and then she uttered a peculiar plaintive cry. She was continually moving her tongue round the interior of her mouth, from time to time thrusting the organ out beyond the lips, and moving it from side to side. The pulse was weak, but regular. The bystanders said that it had been intermittent. The hands and feet were cold. There was constant lachrymation, insomuch that a woman was continually wiping the sufferer's eyelids with a handkerchief. The intensity of symptoms varied greatly; sometimes she would lie quite still for several minutes, and then all at once be seized with a paroxysm of sighing, every now and then exclaiming, "What is it?"

I gave her sesquicarbonate of ammonia, with compound tincture of cardamoms, and hot brandy-and-water, ordered her bed to be warmed, and had her taken up stairs. Having to a certain extent rallied, she managed to go up a steep staircase, assisted by one person. She said that "her face felt very large," and that "all her teeth were coming out." During the night she had very little sleep, her rest being disturbed by dreams that she was falling from a height.

The next day she complained of pain in the lower part of the back and of a numbness in both arms, but these sensations soon subsided, and no ill effects remained. After recovering, she stated that she had not the slightest recollec. tion of what had occurred during two hours-i.e. from half. past twelve till half-past two o'clock.

Remarks.-The above is a good illustration of the effects of a poisonous dose of a preparation of aconite. At first sight the symptoms might have led to a suspicion that hysteria was the cause of the phenomena observed, but the sudden loss of sensation in the branches of the fifth nerve distributed upon the mucous lining of the tongue and the interior of the mouth, followed by vomiting, paralysis of sensation in the arms, \&c., sufficed to suggest that poison had been taken, and that that poison was aconite.

The case also illustrates the uncertainty in the effects produced by the preparations of this substance, and suggests the degree of care which should be taken in prescribing them.

The exact quantity of aconitina contained in the tincture given in the present instance it is impossible to determine, owing to the inadequacy of our tests for that alkaloid; but upon inquiry at the manufacturer's, it was found that the tincture had been prepared by macerating four ounces of the root in one pound of spirit. Of this the patient had taken fifteen minims, a small dose, but one which, from a remark made by Professor Taylor, upon the authority of Dr. Pereira, would appear amply sufficient to occasion dangerous symp. toms.* Of course the strength of the tincture employed must be here taken into account, and this varies extremely; that prepared according to Dr. Fleming's directions consisting of powdered tubers and rectified spirit in the proportion of sixteen ounces (Troy) to sixteen fluid ounces, and there being some five or six other formula known.t Pereira states that

* Taylor on Poisons, (1848,) p. 765 ; and Medical Jurisprudence, (1849,) p. 187 . 